\title{
Récits de vie d'adolescents. Humeur dépressive et figures parentales
}

\author{
Poudat Céline ${ }^{1} \&$ Boulard Aurore ${ }^{2}$ \\ ${ }^{1}$ Université Paris 13, Sorbonne Paris Cité, LATTICE, CNRS (UMR 8094). celine@poudat.fr \\ ${ }^{2}$ Service de Psychologie clinique de l'enfant et de l'adolescent, Université de Liège. Aurore.boulard@ulg.ac.be
}

\section{Problématique et travaux antérieurs}

\subsection{Du développement de la compétence narrative au récit de vie}

Nos premiers travaux sur le développement de la narration chez l'enfant et le pré-adolescent (Boulard, Poudat \& Gauthier, 2012) nous ont permis de mettre en évidence une stabilisation du discours observée à partir de 10 ans.

A partir d'un ensemble de 408 transcriptions du discours oral spontané de 136 enfants et préadolescents âgés de 3 à 13 ans (de la première année maternelle à la deuxième année d'humanité) issus de 12 écoles de la Communauté Française de Belgique, nous avions pu observer comment la compétence narrative se développe pour atteindre une stabilisation vers l'âge de 11 ans (Figure 1). Ces résultats confirmaient de manière empirique que le développement du langage se manifeste bien par un développement rapide du lexique (Bassano et al., 1998). Nos résultats concordant avec la litérature sur le développement du lexique chez l'enfant (Bassano, 1998 ; Clark, 1998) mettaient en évidence un langage enfantin peuplé d'animaux, de mots concrets et mis en scène par des phrases courtes et peu élaborées. Avec le développement cognitif de l'enfant, nous voyions apparaître des énoncés plus complexes, des mots abstraits ainsi que des marqueurs temporels conformément aux observations de Freedman (1990) et Godard \& Labelle (1998). L'enfant commence à mettre en lien certains événements de vie grâce à l'utilisation de subordinations et de compléments circonstanciels, permettant d'ancrer le récit spatialement et temporellement (coomme l'ont déjà bien décrit Hickmann, 2000, Salazar-Orvig, 2004). Nous avions également mis en évidence que l'enfant dès l'âge de 3 ans est capable d'adapter son discours en fonction de son interlocuteur et de la condition dans laquelle il se trouve. Les trois conditions d'enregistrements (seul à la maison avec ses parents, seul avec un chercheur en psychologie et en compagnie de son groupe de pairs) apparaissaient comme significativement différentes. La condition groupe semblait plus régressive en ce qui concerne l'utilisation du vocabulaire et la longueur des énoncés tandis que la condition seul avec un chercheur semblait plus propice à une narration plus développée. Ces résultats convergent avec les observations de Stassen-Berger (2012) selon lesquelles un enfant peut très tôt adapter son discours selon qu'ils interagissent entre eux ou avec leurs enseignants ou leurs parents. Le recouvrement des zones de confiance à partir de 11 ans nous a permis de formuler l'hypothèse d'une stabilisation des acquisitions nécessaires à la narration et plus particulièrement à la narration de soi tout comme le suggère Habermas et Bluck (2000). 


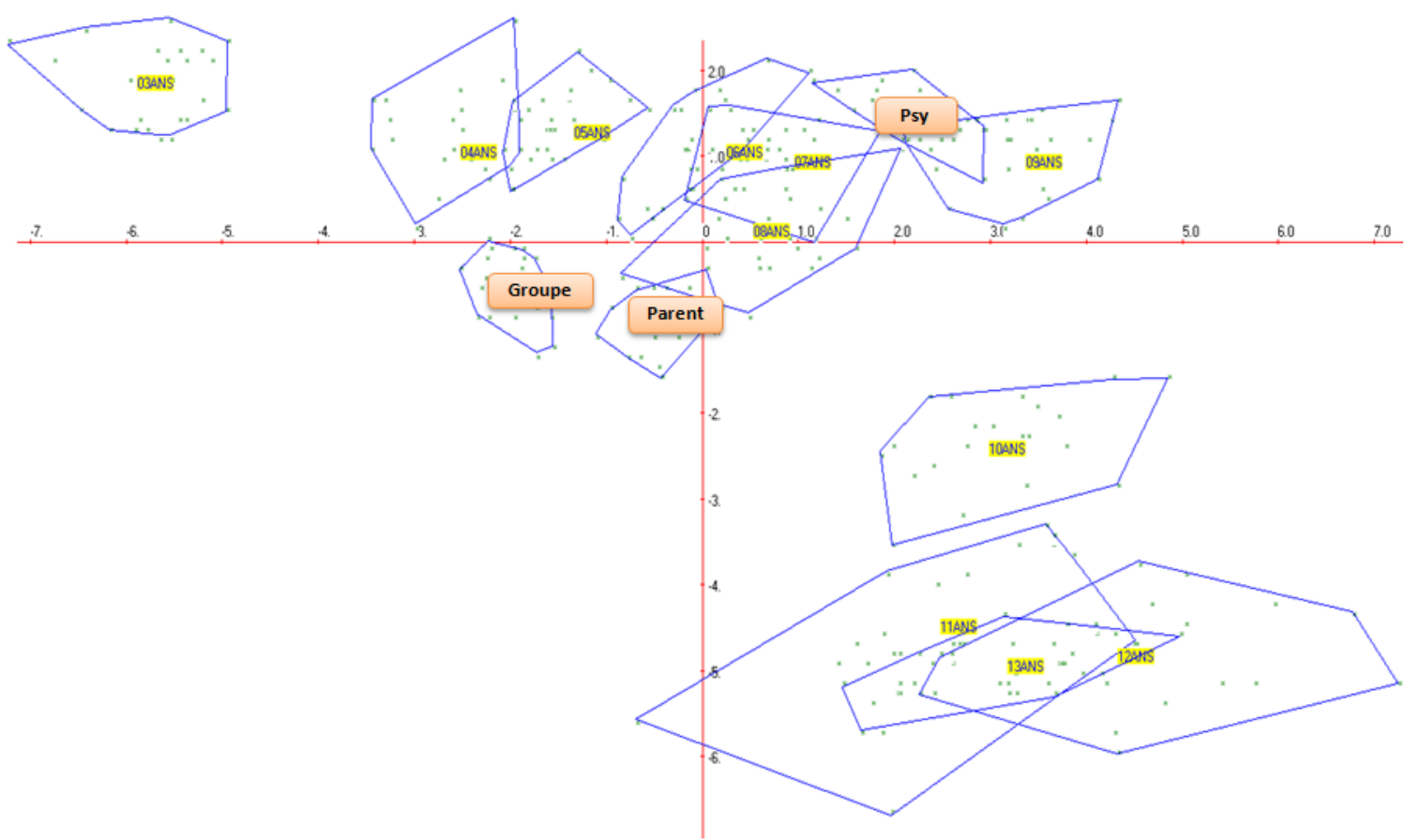

Figure 1: Zones de confiance (enveloppes convexes) autour des 3 situations et des 11 âges observés sur le premier plan factoriel - variables catégorielles illustratives de l'ACP Morphosyntaxe, voir Figure 1 (Boulard, Poudat \& Gauthier, 2012)

Ces résultats nous ont conduit à penser que l'enfant aurait acquis dès cet âge les compétences suffisantes et indispensables à l'élaboration d'un récit, ce qui confirmerait que c'est vers la fin de l'enfance et au début de l'adolescence que l'enfant devient capable de structurer son récit, prérequis nécessaire à la construction d'une identité stable (MacAdams, 2001 ; Mac Lean, 2005).

\subsection{Exploration du récit de vie adolescent}

C'est sur la base de ces résultats que nous avons lancé une première exploration du récit de vie adolescent à partir de 268 récits de vie d'adolescents âgés de 12 à 18 ans, qui nous a permis de mettre en évidence l'effet significatif de l'humeur sur les caractéristiques linguistiques des textes (Poudat \& Boulard, 2014).

Comme le montre la Figure 2 qui ne projette que les catégories significatives sur les axes du premier plan de l'Analyse Factorielle des Correspondances (AFC) appliquée au lexique du corpus, l'humeur dépressive élevée (DEPRESSION 3 sur la carte) s'est avérée potentiellement déterminante dans les discours des adolescents observés.

Le discours des adolescents manifestant des troubles de l'humeur serait significativement corrélé à l'emploi d'un lexique polarisé sur des figures parentales et familiales; très majoritairement de sexe féminin, les sujets positionnés à l'extrémité de ce pôle se racontent à travers le roman familial en se focalisant sensiblement sur des figures féminines (la mère notamment). Caractérisé par un déficit de je, le récit de ces adolescentes semble ainsi plus construit, comme le montre par exemple la présence des temps verbaux du récit (imparfait, plus-que-parfait et passé composé). 


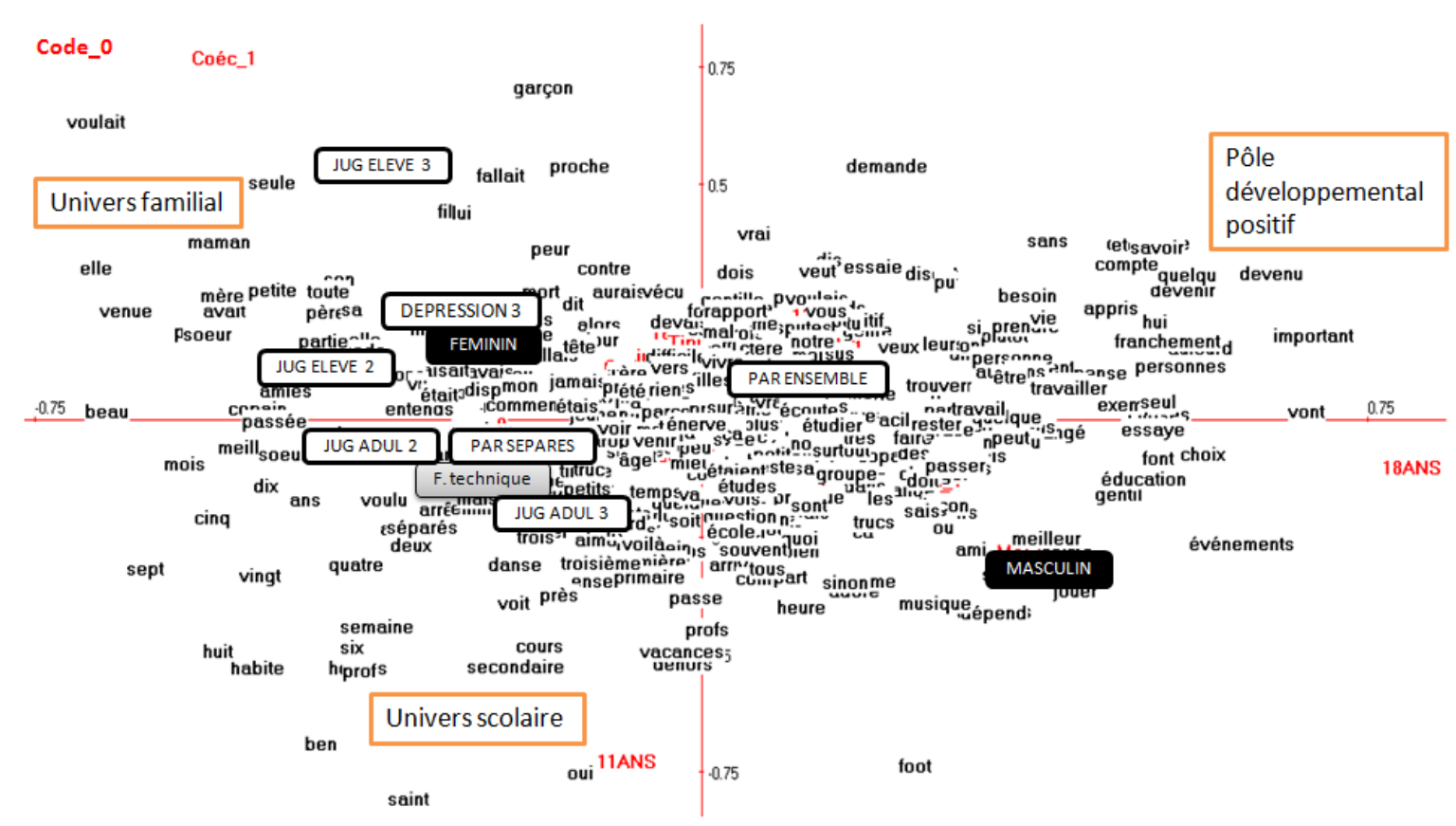

Figure 2. AFC avec projection des variables catégorielles illustratives

Pour Barrera \& Li (1996), même si l'adolescent développe un réseau d'amitiés et revoit ses relations parentales au cours de son adolescence, la famille garde une place prépondérante en termes de ressources, d'attachement. Si celle-ci dysfonctionne, et on peut en faire l'hypothèse, le bien-être de l'adolescent est alors menacé. Nombreuses recherches (Raja et al., 1991; Greenberg et al., 1983) ont d'ailleurs mis en évidence le rôle de la famille et de l'attachement parental sur le bien-être de l'adolescent.

Au vu de ces résultats concordant avec la littérature, nous avons choisi d'explorer l'univers familial des adolescents en lien avec leur humeur; on se concentrera plus spécifiquement sur les représentations des figures parentales dans les discours suivant l'humeur de l'adolescent.

\subsection{Humeur et dépression à l'adolescence}

La dépression à l'adolescence fait depuis quelques années l'objet d'études épidémiologiques. Chez les adolescents, les taux de prévalence varient de 5 à 7\% pour l'épisode dépressif majeur (EDM) avec une prévalence de deux filles pour un garçon (Alvin et Marcelli, 2005). De nombreuses recherches sur la dépression et l'humeur dépressive chez les adolescents mettent en évidence des particularités de cette psychopathologie durant la période spécifique de l'adolescence, à la fois par rapport aux enfants mais également par rapport aux adultes. Une des données les plus stables concernant la dépression à l'adolescence est qu'elle touche deux fois plus de filles que de garçons à partir de 14 ans et que son évolution est également plus rapide chez ces dernières (pour une revue, voir Hankin \& Abramson, 2001).

Parmi les causes probables avancées par les chercheurs pour expliquer cette différence qui se développe à l'adolescence (il n'y a pas de différence de genre concernant la dépression durant l'enfance) et se maintient à l'âge adulte, nous pouvons citer entre autres les ruminations négatives plus importantes chez les filles et les femmes (Nolen-Hoeksema \& Girgus, 1994), la dépendance plus grande aux relations amicales (Cyranowski et al., 2000), les changements hormonaux (Halbreich \& Kahn, 2001), des facteurs génétiques, une image de soi plus négative chez les femmes ainsi qu'une plus grande sensibilité aux événements négatifs (Silberg et al, 1999). Compas et al. (1993) ont proposé d'expliquer la plus grande fréquence de la dépression chez les adolescentes par la plus grande vulnérabilité des filles aux sentiments dépressifs. Elles focaliseraient leur attention sur les affects dépressifs et la symptomatologie dépressive, 
alors que les garçons, au contraire, utiliseraient plutôt les stratégies de détournement de l'attention comme l'agressivité ou l'hyperactivité.

Parmi les facteurs de risque associés à l'étiologie des problématiques adolescentes, les relations familiales ont également été identifiées comme cruciales (Marcotte, 2001). Cette relation doit en plus être considérée comme bi-directionnelle, les conflits familiaux pouvant engendrer la dépression chez un membre de la famille, comme à l'inverse la dépression d'un membre de la famille est un facteur de stress important pour les autres membres (Hammen, 1991). Angold et Costello (1987) expliquent quant à eux la plus grande proportion de filles souffrant d'humeur dépressive par la présence de conflits familiaux.

Les auteurs s'accordent en général pour décrire l'impact plus important de la relation avec la mère sur le bien-être des adolescents, le père étant plus distant, ou en tout cas perçu comme tel par les adolescents.

Un des effets de la dépression, reconnu depuis plusieurs années, est d'affecter la production discursive (Mundt et al., 2007). Les chercheurs ont montré des changements dans la hauteur, la fluidité, la prosodie et dans l'articulation chez les patients déprimés (eg. Darby and Hollinen, 1977; Alpert, Pouget \& Silva, 2001). En outre, les premières études sur les modes de pensées de patients déprimés réalisées par Beck (1976) mettent en évidence que ces patients rapportent davantage d'événements négatifs lorsqu'ils parlent de leur passé. Selon Coyne \& Gotlib (1983), ils ont plus tendance que d'autres à s'attribuer la responsabilité de ces événements négatifs (attributions internes). Ils projettent leur sentiment d'impuissance sur le monde (Seligman, 1975). Cette attribution excessive de la responsabilité des événements devrait se traduire par une utilisation importante du pronom personnel « je » (Mirabel-Sarron, 1992). Bucci \& Freedman (1981) observent d'ailleurs que les patients dépressifs utilisent plus régulièrement le pronom personnel « je » et parlent davantage d'événements passés que les patients du groupe contrôle.

De nombreux articles ont vu le jour ces dernières années sur les récits de vie (MacAdams, 2006, 2008 ; McLean, Pasupathi \& Pals, 2007), leur place dans le champ de la psychologie de la personnalité (Bamberg, 2004 ; Fivush, 2000 ; Thorne, 2000), ainsi que sur le développement et l'émergence de la capacité à construire un récit de vie cohérent (Habermas \& Bluck, 2000). Cependant, peu de chercheurs en psychologie ont étudié à la fois la structure et les propriétés linguistiques du récit auprès d'adolescents souffrant d'humeur dépressive et de dépression, ce qui renforce l'intérêt de notre démarche et son caractère inédit..

Après avoir présenté les données qui fondent cette étude et la méthodologie que nous leur avons appliquée (2.), nous nous concentrerons sur les représentations des figures parentales dans les discours, de leur distribution (3.) à leur mise en discours (4.). Nous proposerons enfin différentes pistes d'analyse à poursuivre (5.)

\section{Données et méthodologie}

\subsection{Corpus et questionnaire}

\subsubsection{Population}

Notre échantillon se compose de 206 adolescents (120 filles et 86 garçons ) âgés de 12 à 18 ans (moyenne $=14,5$ ans) issus de 12 écoles de la communauté française de Belgique. Ils sont répartis en 3 catégories d'âge : 64 adolescents font partie de la catégorie d'âge [12-13], 71 adolescents appartiennent à la catégoire [14-15] et 71 adolescents font partie de la catégorie [16-18]. Les trois filières d'enseignement sont représentées soit $70 \%$ en enseignement général, $21 \%$ issus de l'enseignement technique et $9 \%$ issus de l'enseignement professionnel.

En ce qui concerne les scores de dépression, les résultats obtenus à l'échelle CES-D concordent avec les données épidémiologiques habituelles: $20 \%$ des adolescents de notre échantillon obtiennent un score 
d'humeur dépressive sévère (>24). Parmi ces $20 \%$, les filles y sont deux fois plus nombreuses (33 contre 10 garçons).

Chaque adolescent s'est prêté à un entretien en deux parties.

\subsubsection{Questionnaire}

La première partie de l'entretien consiste en la passation d'un questionnaire comprenant différentes variables. Ce questionnaire a été construit en lien avec la litérature sur l'humeur dépressive et la dépression chez les adolescents. Nous avons choisi d'aborder différents aspects de la vie sociale des adolescents. Différentes informations générales on été récoltées : l'âge, le sexe, le type de filière scolaire (générale, technique ou professionnelle), l'année d'étude et l'option, et le statut des parents (mariés divorcés).

Ensuite, se sont succédées différentes échelles : l'Intimate friendship scale qui mesure le degré d'attachement au meilleur ami et/ou à la meilleure amie; le Parental Bonding instrument (PBI) qui mesure l'attachement de l'adolescent à ses parents (un score est obtenu pour le père et un score pour la mère); une variable mesurant le bien être à l'école et en classe ainsi que le sentiment de se sentir jugé par les pairs et les adultes présents à l'école (professeur, éducateur, préfet, directeur,...). Enfin, l'humeur de l'adolescent est mesurée grâce à l'échelle CES-D. L'échelle CES-D (Center for Epidemiological Studies Depression scale) est un auto-questionnaire comportant 20 items. Chaque réponse est cotée de 0 à 3 . Cette échelle est indiquée pour mesurer la symptomatologie dépressive dans la population générale. Ses propriétés psychométriques ont été testées auprès d'un échantillon d'adolescents francophones (Boulard et al., 2013). Les résultats à la CESD peuvent être catégorisés plusieurs classes (0-16 : aucune symptomatologie; $16-23=$ dépression modérée; 24 et plus= dépression sévère). Morin et al (2011) ont montré l'efficacité des propriétés de dépistage de la CES-D. L'originalité de l'échelle réside en deux points : dans sa méta-analyse des structures factorielles de quatre échelles de dépression, Shafer (2006) montre que la CES-D est la seule échelle à prendre en compte les problèmes interpersonnels. Elle contient également des variables somatiques motivationnelles que d'autres échelles telles que la Beck ou la HRSD ne contiennent pas.

\subsubsection{Corpus}

Le corpus textuel de récits de vie a été constitué sur la base d'une question libre, qui faisait suite au questionnaire, et durant laquelle le chercheur s'est abstenu d'intervenir, afin de laisser l'adolescent construire sa narration. La question Qu'est-ce qui a fait que tu es devenu ce que tu es aujourd'hui ? a été posée à l'adolescent, éventuellement complétée par la question Est-ce qu'il y a des événements positifs ou négatifs qui ont fait que tu es devenu(e) ce que tu es aujourd'hui?

Chaque récit a été enregistré et retranscrit par le chercheur qui a fait passer l'entretien. La durée de l'enregistrement a été standardisée et fixée à 10 minutes (après un testing réalisé lors de pré-tests); on observe au final une moyenne de 950 tokens par récit, et la représentation suivante (Figure 3) lorsqu'on partitionne le corpus suivant les trois catégories d'humeur retenues: 


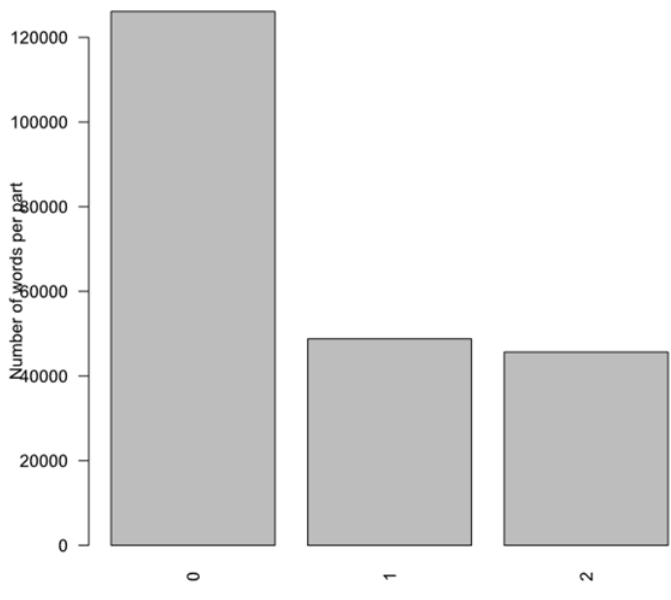

Figure 3. taille de la partition du corpus en nombre de mots suivant le CES-D.

( $\mathrm{o}$ = pas d'humeur dépressive, 1 = humeur dépressive légère à modérée, 2 = humeur dépressive sévère)

\subsection{Méthodes et outils mobilisés}

Les analyses quantitatives ont été réalisées avec le logiciel d'analyse de données numériques et textuelles DtmVic, développé par L. Lebart; nous avons notamment recouru à la méthode ANALEX, qui permet d'explorer et de visualiser le tableau agrégé d'une partition, en l'occurrence, la partition des données suivant les trois catégories humorales considérées. Deux types d'observations ont ainsi été pris en compte : un ensemble d'observations linguistiques que nous avons complété par les 20 variables extraites du questionnaire.

Les analyses qualitatives ont quant à elles été réalisées avec l'outil de linguistique de corpus et d'analyse de données textuelles TXM. Nous avons essentiellement exploité les fonctionnalités de concordance, de recherche de spécificités sur une partition du corpus et de cooccurrences. Deux niveaux de l'analyse linguistique ont pu être mobilisés: le lexique (mots-formes et lemmes), et la morphosyntaxe, appréhendée au moyen de l'analyseur (morpho)syntaxique Cordial (Société Synapse Développement), qui effectue une analyse syntaxique (syntagmatique) des textes et affecte à chaque unité segmentée une catégorie morphosyntaxique.

\section{Spécificités et distributions des figures parentales}

Le questionnaire d'attachement parental soumis à la cohorte nous a d'abord permis d'observer certaines régularités éclairantes, d'autant qu'il faut souligner que ces variables se sont avérées être les plus déterminantes pour spécifier les différences d'humeur: les adolescents ayant obtenu un score d'humeur dépressive élevée (désormais $\mathrm{HD}++$ ) semblent d'abord surprotégés par leurs deux parents (overpère valeur-test $+4,539$; overmère $+2,5$ ) tout en manifestant un déficit de soins maternels (caremère $-4,540$ ); les adolescents d'humeur dépressive modérée (désormais $\mathrm{HD}+$ ) partagent ce sentiment d'étouffement maternel (overmère +2,676) alors que les adolescents qui ne connaissent pas de trouble de l'humeur (désormais HD-) rendent compte au contraire de soins parentaux satisfaisants (caremère $+4,571$; carepère $+2,720)$ sans surprotection.

Ces éléments sont ainsi les premiers à émerger significativement, et comme l'atteste la littérature (e.g. Handa et al., 2009 ; Rey, 1995), la relation de l'adolescent à ses deux parents joue un rôle important sur son humeur.

Si ces données sont éclairantes quant à la manière dont l'adolescent se représente ses relations avec ses parents, les récits de vie dont nous disposons nous permettent d'apporter des éléments de description plus inédits. 
Commençons par présenter quelques données quantitatives, qui font écho aux observations précédentes de manière frappante: la mère et le père sont ainsi significativement plus représentés dans les récits des adolescents $\mathrm{HD}++$; mieux, mère et père sont parmi les tout premiers mots à émerger pour caractériser les discours de ces adolescents lorsqu'on calcule les spécificités des trois sous-ensembles. On note également que maman est plus spécifique aux $\mathrm{HD}+$ et $\mathrm{HD}++$, ce qui va dans le sens du score overmère élevé observé dans ces deux groupes.

Soulignons enfin que le mot parents participe à la spécification négative des $\mathrm{HD}++$, ce qui montre bien que les sujets concentrent leur récit sur les personnages parentaux plutôt que sur les parents en tant qu'entité et apport.

\begin{tabular}{|l|l|l|l|l|l|l|l|}
\hline Unités & F & f_HD- & Score & f_HD+ & Score & f_HD++ & Score \\
\hline mère & 324 & 149 & -4.4882 & 81 & 0.9246 & 94 & 3.6167 \\
\hline père & 334 & 180 & -0.9096 & 64 & -0.971 & 90 & 2.4281 \\
\hline papa & 241 & 125 & -1.2603 & 53 & -0.2856 & 63 & 1.6062 \\
\hline maman & 322 & 174 & -0.8565 & 77 & 0.6255 & 71 & 0.5295 \\
\hline parents & 556 & 331 & 0.8533 & 129 & 0.5475 & 96 & -1.6241 \\
\hline
\end{tabular}

Tableau 1. Spécificités des figures parentales dans les trois groupes - TXM

La Figure 4 est ainsi éloquente: près de $80 \%$ des discours des HD++ contiennent au moins une occurrence des figures maternelles (mère ou maman) et paternelles (père ou papa) alors que les HD- ne les mentionnent explicitement que dans un texte sur deux. A contrario, près de $70 \%$ des HD- évoquent leur famille (présence d'au moins un lemme famille ou familial) tandis que les HD+ et HD++ n'en font mention que dans un discours sur deux. 


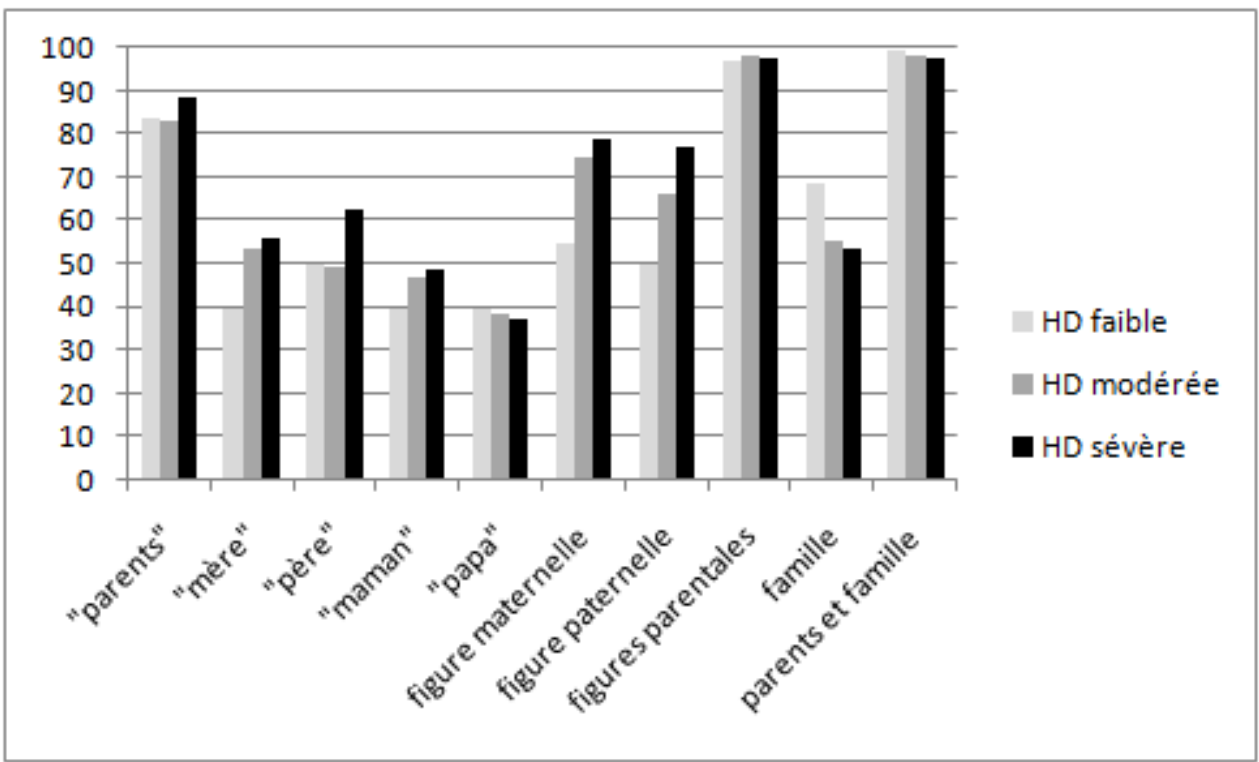

Figure 4. Présence des figures parentales d'une catégorie humorale à l'autre

Il est de toute évidence indiscutable que l'adolescent se définit relativement à ses parents puisque 97\% des discours en contient au moins une mention - et 99\% des HD- évoquent leur famille lorsqu'ils ne mentionnent pas leurs parents.

A noter que seuls trois sujets n'évoquent ni leurs parents ni les lexèmes famille ou familial: si le premier ne se définit que relativement à son parcours scolaire (036), les deux autres, qui racontent tout de même leur fratrie ou un grand-père, éprouvent des difficultés avec la question:

Je ne croyais pas que ça allait être si dur des questions comme ça. (107, HD-, extrait)

Je ne sais pas ben il n'y a rien mes relations se sont bien passées je ne sais pas ben voilà quand on est en primaire on ne sait pas grand-chose et quand on rentre en secondaire ça change quoi voilà je ne sais pas non avant j'étais timide mais maintenant plus je ne sais pas ça se passe un peu bien à l'école je parle beaucoup je ne sais pas quoi dire je n'ai rien à dire j'ai beaucoup de frères et sœurs j'en ai sept j'ai trois frères et quatre sœurs quand on veut étudier ou quoi ben ça parle quoi (130, HD++, réponse intégrale)

\section{Les personnages maternel et paternel en discours}

Analysons maintenant la manière dont sont mises en discours les figures maternelles et paternelles.

\subsection{Père et mère, papa et maman}

On note d'abord que les sujets ont le choix entre les paires papa et maman, et père et mère, qui marquent des frontières distinctes: papa et maman renvoient à l'intérieur, puisque c'est ainsi que le sujet désigne ses parents dans l'intimité de la cellule familiale (ou avec ses proches en général); père et mère renvoient à l'extérieur, puisque c'est avec ces lexèmes que l'individu désigne généralement ses parents dans le monde extérieur. Soulignons que nous n'avons relevé aucune corrélation significative de ces paires avec les âges des adolescents.

Ces oppositions intérieur/extérieur, proche/distant sont particulièrement apparentes dans les discours lorsque l'adolescent alterne entre les deux systèmes pour désigner le même parent:

ma maman m'a fait ce qui a fait que je suis aujourd'hui vs avant, je ne me disputais pas vraiment avec ma mère mais depuis que mes parents sont divorcés... (081, HD++) 
et ma mère par contre elle a tout le temps eu des copains différents donc c'est assez marrant par contre (...) ma maman maintenant elle est avec quelqu'un depuis un an c'est un miracle (020, HD-)

Avec le jeu des séparations, des nouveaux couples et des beaux-parents, certains adolescents peuvent d'ailleurs être amenés à distinguer formellement le père du papa, le papa renvoyant aux soins paternels, à "l’homme proche de moi qui m'a élevé(e)".

J'ai eu un petit problème quand j'étais jeune parce que mon père est parti quand j'avais trois mois. (...) j'ai vraiment un blocage par rapport à mon père. Je ne vois plus du tout mon père. (...) Ma maman s'est mariée avec un deuxième homme qui est le papa de ma petite soeur (...) je lui ai demandé si je pouvais l'appeler papa. Quand il est parti, il est encore reparti donc j'avais de nouveau plus de papa. (095, HD-)

Mon père biologique je le vois par moment mais il travaille la plupart du temps et donc je le vois rarement. Je n'ai jamais dormi chez lui. Et ça ne me fait pas grand-chose parce qu'il y a mon beau-père. C'est un peu mon papa. Enfin c'est lui qui m'a élevée. (102, HD-)

Enfin, dans certains cas et certaines situations, l'adolescent ressent pour sa mère une plus grande proximité qui le conduit à former une paire déséquilibrée "ma maman et mon père":

C'est ma maman et mon père qui m'ont donné dans cette vie. (030, HD-)

quand mon père a trompé ma maman je ne sais pas, ça m'a aussi fait changer enfin je ne sais pas, j'ai eu, je ne sais pas mais à partir de ce moment-là j'avais moins confiance en moi je ne sais pas pourquoi $(159, \mathrm{HD}++)$

Si l'ensemble des sujets privilégient père à papa, les formes maman et mère semblent relativement équidistribuées; les HD- semblent privilégier maman à mère (Tableau 1), mais l'examen des contextes et des cooccurrents des deux formes ne met en évidence que des différences de proximité telles qu'évoquées précédemment (e.g. gentils, câlin (+2) autour de maman).

\subsection{Examen des cooccurrents}

L'observation des cooccurrents s'est avéré plus pertinente et plus efficace que l'étude des concordances et des régularités syntagmatiques autour des formes considérées; en effet, aucune régularité vraiment significative sur le plan quantitatif n'a pu être mise au jour avec la dernière méthode, ce qui n'est pas surprenant du fait de la nature même des données. Le récit de vie de l'adolescent est loin de la routine discursive et la diversité des histoires personnelles, des parcours et des horizons socioculturels va de pair avec des variations linguistiques sensibles, même si la période de l'adolescence stabilise le lexique puisque les sujets évoluent dans des environnements comparables (vie avec les parents, scolarisation, activités extra-scolaires) qui ancrent les discours.

A contrario, la méthode des cooccurrences, qui prend en compte une fenêtre contextuelle plus large, nous a permis d'observer certaines régularités discursives et énonciatives que nous restituons ici.

Ainsi, l'examen des cooccurrents des formes mère et maman, et père et papa, nous a permis toutefois de mettre au jour des différences significatives entre les $\mathrm{HD}++$ et les autres groupes en ce qui concerne spécifiquement leur usage de mère et père. Dans les autres groupes, mère et maman, et père et papa ont en effet des cooccurrents comparables: les deux formes sont d'abord très fortement corrélées avec le déterminant possessif de première personne (ma maman, ma mère, mon papa, mon père), suivi de la figure paternelle ou maternelle associée. On observe quelques variations entre les deux formes, notamment concernant l'usage de la préposition chez, significativement corrélée à maman (+7 HD-, +6 $\mathrm{HD}+)$ et à papa (+8 HD-, $+6 \mathrm{HD}+)$, ce qui fait écho aux différences de proximité et de frontières évoquées dans la section précédente.

De leur côté, les $\mathrm{HD}++$ racontent davantage leur mère: mère est d'abord corrélée à l'anaphorique elle $(+7)$ avant d'être associée à père $(+6)$, puis encore à mère $(+4)$, ce qui signale bien la présence d'un développement autour du personnage de la mère: 
Il s'est marié avec ma mère et puis il l'a laissée tomber puis ma mère et moi on est parti vivre avec ma petite soeur (193, HD++).

$\mathrm{Au}$ vu des cooccurrents, on pourrait penser qu'il en va de même pour le père, également fortement corrélé à d'autres occurrences de père dans la même fenêtre contextuelle $(+5)$ et à l'anaphorique $i l(+4)$ chez les $\mathrm{HD}++$. Mais il en va finalement différemment puisque les père intercorrélés cadrent le récit dans la lignée familiale plutôt qu'ils ne signalent une thématisation du père dans le récit:

il y a eu le père de mon père la soeur de mon père (008, HD++)

les parents de mon père ils habitent juste à côté et puis si mon père va être déprimé (108, HD++)

On retrouve d'ailleurs ce phénomène dans les discours de l'ensemble des adolescents; chez les HD-, père est corrélé à grand-père, côté (du côté de mon père)...

Si comme l'atteste la littérature, la mère joue un rôle crucial sur le bien-être des adolescents, il n'en reste pas moins qu'elle incarne un personnage clé, et bien thématisé dans le discours de l'adolescent dépressif.

L'examen des cooccurrents morphosyntaxiques des figures parentales permet de préciser ces éléments de manière intéressante: la mère et le père des HD++ sont en effet d'abord associés aux temps imperfectifs de l'imparfait et du plus-que-parfait $(+4)$, au contraire des autres groupes, qui racontent plutôt leurs parents au passé composé, premier temps verbal associé. La différence est frappante, puisque le passé composé a cette particularité de raconter un événement passé qui a une répercussion dans le présent, et donc sur le sujet:

ou sinon du côté de ma mère ma mère a rencontré quelqu'un qui est tout seul donc voilà (019, HD-)

Quand mon père est parti, quand il a poussé ma mère contre le bord de table, ils se sont disputés c'est quand il est parti qui fait que j'ai changé de caractère, j'ai été plus violente et puis comment dire. (136, HD-)

En revanche, l'imparfait, et son homologue composé le plus-que-parfait, inscrivent le récit dans un passé figé puisque les deux temps ne peuvent sans temps perfectif associé faire progresser la narration:

Alors notre grand-mère, on va dire qu'elle ne nous aimait pas trop et elle était mariée. Et ma mère, elle n'était pas, n'avait pas un lien très fort avec son père et voilà. Et alors ma mère elle avait un petit appart où on dormait ensemble, voilà. Ma grand-mère, ma mère, ma grand-mère, pour qu'elle s'occupe de nous, il fallait que ma mère elle paie. Alors un jour, ma mère les gens lui parlaient de de rencontres, d'un site de rencontres alors elle est partie là. (193, HD++)

Il y avait aussi mon père qui essayait un peu de l'aider vu que ma mère s'en occupait mais ne regardait pas tout ce qu'elle faisait à l'école et tout ça, c'est pas sa fille mais il avait vu que je tenais à elle. Il y avait aussi ma grande soeur qui a vingt-six ans qui tient un bar au casino, elle elle avait quelques soucis avec mon père donc (201, HD++)

Les parents de ma mère je ne les ai pas connus parce que quand ma mère était jeune elle se faisait enfin elle a eu des abus sexuels (204, HD++).

Les verbes conjugués à l'imparfait et fléchis à la troisième personne du singulier sont en outre les formes verbales les plus spécifiques des $\mathrm{HD}++(+5,6)$, tandis que c'est bien le passé composé qui est le temps verbal le plus caractéristique des HD- $(+4,8)$. Cette remarque pourrait faire écho au fait que l'expérience du temps est connue pour être altérée dans la dépression. Le temps est expérimenté comme "arrêté", sans futur où le passé émerge du présent (Kuhs, 1991). C'est pour cette raison que le temps, et les changements, semblent avoir fait une halte chez les patients dépressifs qui sont parfois décrits comme vivant dans le passé (Habermas, 2008). 


\section{Conclusion et perspectives}

Cette première recherche sur les liens entre l'humeur dépressive et le langage s'est centrée sur les figures d'attachement parentales, premiers paramètres à jouer un rôle déterminant pour spécifier les différences d'humeur de l'adolescent. Si 97\% des adolescents évoquent leurs parents dans leurs récits de vie, les adolescents dépressifs se concentrent sur la figure maternelle, personnage fortement thématisé dans leur récit - au demeurant caractérisé par un déficit de je, marquant un certain effacement de soi; l'analyse des cooccurrents des figures parentales nous a permis de mettre au jour des phénomènes énonciatifs plus généraux et d'observer un certain figement du récit, avec un usage significatif des temps imperfectifs. On a ainsi pu observer des juxtapositions de verbes conjugués à l'imparfait, comme si l'adolescent était luimême suspendu au passé de son récit, tandis que les adolescents sans troubles de l'humeur, plus raccordés au présent, recourent d'abord au passé composé.

Il conviendra maintenant d'investiguer la question des événements associés aux troubles de l'humeur, mais nous avons vu que l'entreprise était complexe étant donné la diversité des récits et des adolescents, entrainant des variations telles que les recherches de concordances autour d'une forme ou d'un prédicat sont peu efficaces. En outre, le récit peut être fragmentaire ou elliptique: il serait bien sûr naïf de le considérer comme le reflet fidèle de la réalité d'une vie, d'autant que nos données sont des productions orales spontanées, qu'il pourrait d'ailleurs être intéressant de comparer à des récits écrits. De la même manière, notre démarche nous semble susceptible d'intéresser les travaux actuels menés dans le champ de la psycholinguistique, qui concentrent leurs efforts sur des phénomènes linguistiques et discursifs très fins (observation du chaînage référentiel, des types de discours direct et indirect mobilisés, e.g. Canut 2000, ou des marqueurs de cohésion textuelle dans la production des récits, e.g. Salazar-Orvig 2004); notre approche quantitative, plus structurelle et plus globale, pourrait s'articuler et se nourrir de ces travaux afin d'approcher de manière plus pertinente et plus fine la manière dont le récit se construit et se stabilise.

Notons également que l'examen des effets de l'humeur sur le récit de vie fait apparaitre deux biais: (i) on a ainsi remarqué que les adolescents ne connaissant pas de troubles de l'humeur peuvent avoir du mal à élaborer un récit et produire un discours au fil de l'eau, parfois entrecoupé de questions à la psychologue; tandis que (ii) les adolescents très dépressifs peuvent être mutiques, ponctuant des bribes elliptiques de "je ne sais pas", "je ne vois pas" ou "je n'ai rien à dire". En revanche, on a pu observer, indépendamment de la question des troubles de l'humeur, que certains sujets produisent d'emblée un récit construit et semblent éprouver un désir ou un besoin pressant de raconter leur histoire - à tel point que certains récits ont dû être tronqués, avec la contrainte de durée de dix minutes. C'est sur ces productions que nous pensons particulièrement approfondir la question de ce qui fait événement, ainsi que la structure et l'organisation du récit. Nous avons ainsi remarqué que la question de la séparation parentale était généralement posée en début de récit. Nous notons également que le fait que les parents soient toujours ensemble est mentionné comme un événement, également en début de réponse, ce qui semble indiquer la présence d'une trame sous-jacente - la réponse serait organisée et contrainte par les normes du genre discursif du récit de vie.

Mes parents n'ont pas divorcé. Je ne sais pas vraiment comment expliquer. J'ai une petite soeur, elle a huit ans (015, HD-, début de réponse)

Déjà mes parents se sont séparés il y a six ans donc ce n'était pas amusant le moment même mais six ans après il a y beaucoup de points positifs (024, HD-, début de réponse)

Je ne vois pas ben pour l'instant il s'est passé plein de choses donc par exemple il y a la séparation entre mes parents $(045, \mathrm{HD}+$, début de réponse)

Peut-être que mes parents sont pas divorcés, que il n'y a pas eu de problème dans la famille enfin tous des trucs comme ça (085, $\mathrm{HD}+$, début de réponse)

Les relations que j'ai, l'éducation, voilà, alors là je n'arrive pas, je ne sais pas, eh bien mes parents divorcés, mon père s'est remarié. (070, $\mathrm{HD}++$, début de réponse) 
Une réflexion approfondie sur ce qui fait événement dans une vie et dans un récit de vie devra de toute évidence être poursuivie. Ainsi, pour Filipp (1990), les événements de vie nécessitent ou adviennent en même temps que des changements comportementaux. Les accidents de la vie (maladie, deuil, divorce) sont des événements stressants pour l'individu qui va devoir réorganiser ses relations sociales et être parfois confronté à des changements de valeurs qui peuvent le perturber un certain temps. Il est donc légitime que ces ruptures soient rapportées par l'adolescent en tant qu'événements les définissant.

Enfin, d'autres pistes restent à approfondir, notamment les effets de l'exclusion par le groupe de pairs et du jugement perçu par les adolescents sur la narration de soi. D'autres thématiques spécifiques à l'adolescence, comme l'univers scolaire, les activités extra-scolaires, la capacité de se projeter dans l'avenir mériteraient également d'être étudiées plus avant.

Si les critères du DSM IV ou de la CIM-10 sont assez clairs pour appréhender le phénomène dépressif dans sa globalité, diagnostiquer ou étudier la dépression chez des enfants ou des adolescents est moins aisé. Cette difficulté est due principalement au fait que les manifestations de cette pathologie diffèrent fortement chez l'enfant et l'adolescent par rapport à l'adulte (Avenevoli et al., 2008; Carlson, 2000 ; Cicchetti \& Toth, 1998). Des échelles de mesures, avec des propriétés psychométriques satisfaisantes, sont utilisées pour appréhender cette pathologie dans la population tout-venant. Cependant, elles n'incluent pas la vision subjective qui est centrale dans la dépression et ce, particulièrement à l'adolescence (Lachal et al., 2012). Or cette subjectivité, apparaît dans les récits de vie. Le récit nous montre comment l'adolescent réinterprète et évalue les événements vécus (cfr Bruner, 1990). Si notre recherche s'inscrit dans la continuité des travaux sur les liens entre les récits de vie et la psychopathologie (Habermas et al, 2008 ; Philips, 2003 ; Fivush et al., 2003), l'utilisation des méthodes ADT nous permet de faire un pont entre l'étude qualitative des récits de vie et l'étude quantitative, épidémiologique grâce à la projection de variables métriques telles qu'un score de dépression, d'attachement parental... sur le vocabulaire du corpus étudié. Elle permet donc de visualiser l'adéquation des résultats issus de deux méthodologies complémentaires. 


\section{Références bibliographiques}

Alpert, M., Pouget, E. R., \& Silva, R. R. (2001). Reflections of depression in acoustic measures of the patient's speech. Journal of affective disorders, 66(1), 59-69.

Alvin, P.,Marcelli, D., Archambeaud, M-P., Bader-Meunier, B., Baruchel,A., \& coll. (2005). Médecine de l'adolescent. Paris,Masson.

Angold, A., Costello, E.J., \& Worthman, M. (1998). Puberty and depression: the roles of age, pubertal status and pubertal timing. Psychological Medicine, 28, 51-61.

Avenevoli, S., Knight, E., Kessler, R. C., \& Merikangas, K. R. (2008). Epidemiology of depression in children and adolescents. In J. R. Z. Abela, \& B. L. Hankin (Eds.), Handbook of depression in children and adolescents (pp. 6-32). New York, NY: Guilford Press.

Bamberg, M. (2004). Narrative discourse and identities. In JC. Meister, T. Kindt, W. Schernus \& M. Stein (Eds.), Narratology beyond literary criticism (pp. 213-237). Berlin \& New York: Walter de Gruyter.

Bassano, D. (1998). Sémantique et syntaxe dans l'acquisition des classes de mots: l'exemple des noms et des verbes en français. Langue française, 26-48.

Bassano, D., Maillochon, I., \& Eme, E. (1998). Developmental changes and variability in the early lexicon: a study of French children's naturalistic productions. Journal of Child Language, 25(3), 493-531.

Boulard, A., Gauthier, J. M., \& Born, M. (2013). Étude des propriétés psychométriques de l'échelle CES-D sur un échantillon d'adolescents francophones scolarisés. L'Encéphale.

Boulard, A., Poudat, C., \& Gauthier, J. M. (2012). Des mots pour se dire: Développement de la fonction narrative chez l'enfant. In Actes des $11^{e} J A D T, 203-214$.

Bruner J. (1990). Acts of meaning. Cambridge: Harvard University Press.

Bucci, W., \& Freedman, N. (1981). The language of depression. Bulletin of the Menninger Clinic 45, 4, 334-358.

Canut, E. (2011). De la valeur fondamentale de l'activité narrative dialogique dans la structuration du langage de l'enfant non encore lecteur : instances énonciatives et appropriation du discours indirect. In Actes du XII Simposio Internacional de Comunicación Social, 379-384.

Carlson, G.A. (2000). The challenge of diagnosing depression in childhood and adolescence. Journal of affective disorder, 61, S3-S8.

Cicchetti, D., \& Toth, S.L. (1998). The development of depression in children and adolescents. American Psychologist, 53(2), 221-241.

Clark, E. V. (1998). Lexique et syntaxe dans l'acquisition du français. Langue française, 49-60.

Compas, B.E., Orosan, P.G., \& Grant, K.E. (1993). Adolescent stress and coping : Implications for psychopathology during adolescence. Journal of Adolescence, 16, 331-349.

Coyne, J.C., \& Gotlib, I.H. (1983). The role of cognition in depression: A critical appraisal. Psychological Bulletin, 94(3), 472-505.

Cyranowski, J., Frank, E., Young, E., \& Shear, K. (2000). Adolescent onset of the gender difference in lifetime rates of major depression : A theorical model. Archives of General Psychiarty, 57, 21-27.

Darby, J. K., \& Hollien, H. (1977). Vocal and speech patterns of depressive patients. Folia Phoniatrica, 29, $279-291$.

Fivush, R. (2008). Remembering and reminiscing : How individual lives are constructed in family narratives. Memory Studies, 1(1).

Fivush, R., Hazzard, A., McDermott Sales, J., Sarfati, D., \& Brown, T. (2003). Creating coherence out of chaos? Children's narratives of emotionally positive and negative events. Applied Cognitive Psychology, 17(1), 1-19.

Freedman, W.J. (1990). Children's representations of the pattern of daily activities. Child Development, 61(5), 13991412. 
Godard, L., \& Labelle, M. (1998). Le développement de la localisation dans le temps chez des enfants de 5 à 9 ans de milieux socio-économiques différents. L'Année psychologique, 98(2), 233-270.

Greenberg, M. T., Siegel, J. M., \& Leitch, C. J. (1983). The nature and importance of attachment relationships to parents and peers during adolescence. Journal of Youth and Adolescence, 12(5), 373-386.

Habermas, T., Ott, L.M., Schubert, M., Schneider, B., \& Pate, A. (2008). Stuck in the past: negative bias, explanatory style, temporal order, and evaluative perspectives in life narratives of clinically depressed individuals. Depression and Anxiety, 25(11), 121-132.

Habermas, T., \& Bluck, S. (2000). Getting a life: The emergence of the life story in adolescence. Psychological Bulletin, 126(5), 748-769.

Halbreich, U., \& Kahn, L. (2001). Role of estrogen in the aetiology and treatment of mood disorders. CNS Drugs, 15, 797-817.

Hammen, C. (1991). Generation of stress in the course of unipolar depression. Journal of Abnormal Psychology, $100(4), 555-561$.

Hankin, B.L., \& Abramson, L.Y. (2001). Development of gender differences in depression : An elaborate cgnitive vulnerability-transactional stress theory. Psychological Bulletin, 127(6), 773-796.

Hickmann M., (2000). Le développement de l'organisation discursive. In Kail M. et Fayol M., L'acquisition du langage. Le langage en développement. Au-delà de trois ans. Paris : PUF. 83-115

Lachal, J., Speranza, M., Schmitt, A., Spodenkiewicz, M., Falissard, B., Moro, M. R., \& Revah-Levy, A. (2012). Depression in adolescence: from qualitative research to measurement. Adolescent Psychiatry, 2(4), 296-308.

Marcotte, D. (2001). L'influence du style parental, de la dépression et des troubles du comportement sur le risque d'abandon scolaire. Revue Française de l'Education, 27(3), 687-712.

McAdams, D. P. (2008). Personal narratives and the life story. In John, O., Robins, R., \& Pervin, L. A., Handbook of personality: Theory and research ( $3^{\text {rd }}$ edition). NY: Guilford Press. 242-262.

McAdams, D.P., \& Pratt, M.W. (2006). A new big five: Fundamental principles for an integrative science of personality. American Psychologist, 61, 204-217.

McAdams, D.P. (2001). The psychology of life stories. Review of General Psychology, 5(2), 100-122.

McLean, K.C., Pasupathi, M., \& Pals, J.L. (2007). Selves creating stories creating selves: A process model of selfdevelopment. Personality and Social Psychology Review, 11(3), 262-278.

McLean, K.C. (2005). Late identity development: Narrative meaning making and memory telling. Developmental Psychology, 41, 683-691.

Mirabel-Sarron, C. (1992). Analyse des discours de patients déprimés. Psychologie Française, 37(3-4), $277-289$.

Morin, A.J.S., Moullec, G., Maïano, C., Layet, L., Just, J.-L., \& Ninot, G. (2011). Propriétés psychométriques du Center for Epidemiologic Studies Depression Sclae (CES-D) sur un échantillon français d'adultes cliniques et non-cliniques. Epidemiology and Public Health.

Mundt, J. C., Snyder, P. J., Cannizzaro, M. S., Chappie, K., \& Geralts, D. S. (2007). Voice acoustic measures of depression severity and treatment response collected via interactive voice response (IVR) technology. Journal of Neurolinguistics, 20(1), 50-64.

Nolen-Hoeksema, S., \& Girgus, J. (1994). An integrative model for the emergence of gender differences in adolescence. Journal of Research on Adolescence, 4, 424-443.

Phillips, J. (2003). Psychopathology and the narrative self. Philosophy, Psychiatry, \& Psychology, 10(4), 313-328.

Poudat, C. et Boulard, A. (2014). Exploration du récit de vie d'adolescents. In Actes des $12^{e}$ JADT (à venir).

Raja, S. N., McGee, R., \& Stanton, W. R. (1992). Perceived attachments to parents and peers and psychological wellbeing in adolescence. Journal of youth and adolescence, 21(4), 471-485.

Salazar Orvig, Anne (Ed.) (2004) La cohésion chez l'enfant, CALAP, $n^{\circ} 24$.

Seligman, M.E. (1975). Depression and learned helplessness in man. Journal of Abnormal Psychology, 84(3), 228238. 
Shafer, A.B. (2006). Meta-analysis of the factor structures of four depression questionnaires: Beck, CES-D, Hamilton, and Zung. Journal of Clinical Psychology, 62(1), 123-146.

Sheeber, L., Hops, H., Alpert, A., Davis, B., \& Andrews, J. (1997). Family Support and Conflict: Prospective Relations to Adolescent Depression. Journal of Abnormal Child Psychology, 25(4), 333-344.

Silberg, J., Pickles, A., Rutter, M., Hewitt, J., Simonoff, E., Maes, H., et al. (1999). The influence of genetic factors and life stress on depression among adolescent girls. Archives of General Psychiatry, 56, 225-232.

Stassen-Berger, K. (2012). Psychologie du développement. Louvain : De Boeck.

Thorne, S. (2000). Data analysis in qualitative research. Evidence Based Nursing, 3, 68-70. 